\title{
An Efficient Multi-Vehicle Routing Strategy for Goods Delivery Services
}

This paper was downloaded from TechRxiv (https://www.techrxiv.org).

\section{LICENSE}

CC BY 4.0

SUBMISSION DATE / POSTED DATE

$26-04-2021 / 30-04-2021$

CITATION

Nguyen, Linh (2021): An Efficient Multi-Vehicle Routing Strategy for Goods Delivery Services. TechRxiv. Preprint. https://doi.org/10.36227/techrxiv.14481702.v1

DOI

10.36227/techrxiv.14481702.v1 


\title{
An Efficient Multi-Vehicle Routing Strategy for Goods Delivery Services
}

\author{
Duy Le ${ }^{1}$, Ying Men ${ }^{1}$, Yunkang Luo ${ }^{1}$, Yixuan Zhou ${ }^{1}$ and Linh Nguyen ${ }^{2}$
}

\begin{abstract}
The paper addresses the problem of efficiently planning routes for multiple ground vehicles used in goods delivery services. Given popularity of today's e-commerce, particularly under the COVID-19 pandemic conditions, goods delivery services have been booming than ever, dominated by small-scaled (electric) bikes and promised by autonomous vehicles. However, finding optimal routing paths for multiple delivery vehicles operating simultaneously in order to minimize transportation cost is a fundamental but challenging problem. In this paper, it is first proposed to exploit the mixed integer programming paradigm to model the delivery routing optimization problem (DROP) for multiple simultaneouslyoperating vehicles given their energy constraints. The routing optimization problem is then solved by the multi-chromosome genetic algorithm, where the number of delivery vehicles can be optimized. The proposed approach was evaluated in a realworld experiment in which goods were expected to be delivered from a depot to 26 suburb locations in Canberra, Australia. The obtained results demonstrate effectiveness of the proposed algorithm.
\end{abstract}

\section{INTRODUCTION}

Good delivery service is an essential part of a distribution and transportation system, which has significant impact on today's e-commerce. And given the COVID-19 pandemic crisis with travelling restrictions and social distancing implementations, a couple of online shopping and goods delivery services has been booming than ever [1]. Though variety of goods delivery services was considered a long time ago [2], recent advancement of technologies has truly created flexibility of those services. For instance, by using mealordering platforms such as "Uber Eats" and "Deliveroo", one can make their dinner much more flexible and time-efficient. However, price of goods ordered by online shopping heavily depends on transportation costs. That is, there is a need to reduce cost of goods delivery services. To partly address the issue, recently, researchers and scientists around the globe have paid considerable attention on developing autonomous ground vehicles [3] and unmanned aerial vehicles [4] for this purpose. Nevertheless, there are concerns about safety and reliability of the unmanned goods delivery services [5]. On the other hand, delivering goods to customers on time, particularly food, is highly critical [1]. Therefore, nowadays most of delivery companies prefer using small-scaled vehicles, e.g. electricity-assisted cargo bicycles, to hiring traditional transportation methods, e.g. delivery trucks, especially delivering

\footnotetext{
${ }^{1}$ Duy Le, Ying Men, Yunkang Luo and Yixuan Zhou are with College of Engineering \& Computer Science, The Australian National University, Canberra ACT 0200, Australia $\{$ u6923736, u7037272, u6931202u6327833\}@anu.edu.au

${ }^{2}$ Linh Nguyen is with School of Engineering, Information Technology and Physical Sciences, Federation University Australia, Churchill VIC 3842, Australia l.nguyen@federation.edu . au
}
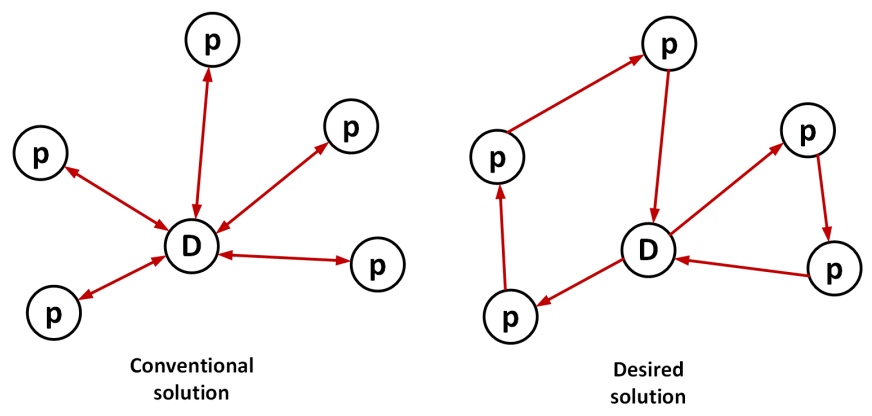

Fig. 1: A demonstration of strategies for vehicles in goods delivery services. A vehicle delivers goods to one customer (left) versus multiple customers (right) at each route, where $\mathrm{D}$ is a depot and $\mathrm{p}$ is a purchaser.

freight in urban areas where traffic conditions are always crowed and complicated. For instance, analysis conducted by Sheth et al. [6] shows that delivery costs by using electricityassisted cargo bicycles are more cost-effective that those of using delivery trucks in regions that are in close proximity to a distribution center (also known as depot) since there are dense neighbourhood residential units and relatively low delivery volumes.

Although the small-scaled delivery vehicles have advantages and popularity as compared with other types of delivery modes, they are constrained by their relatively smaller loader capacity and limited battery life, which prevent them from long operating time and travelled distances. In other words, in order to maximize effective operations of those smallscaled delivery vehicles, it is expected to find their optimal routing paths given delivery requests and their constraints in a specific regions. Though the delivery routing optimization problem (DROP) can be mathematically formulated, optimally solving it is an NP-hard issue [7], [8], [9]. In fact, the DROP is deemed similarly to the travelling salesman problem (TSP). In literature, there is a rich library of approaches proposed to address the TSP, including those based on cutting planes [10], tabu search [11], branch-andbound [12], and neural network [13]. The methods can be divided into two main categories: (1) exact algorithms and (2) near-optimal algorithms. While the exact approaches use integer linear programming techniques with additional constraints, the near-optimal algorithms can efficiently find approximate solutions with guaranteed bounds.

In practice, delivery corporations always run multiple delivery vehicles in the same time, which is contrary to a single TSP. Hence, the DROP becomes finding optimal 
routing paths for multiple vehicles simultaneously. In this paper, in order to mathematically formulate the DROP for multiple delivery vehicles (mDROP), we propose to exploit the mixed integer programming model. It is noted that the optimization problem is constrained by the vehicle resources such as energy and capacity. We then employ the genetic algorithm [14] to efficiently solve the mDROP problem. A demonstration of the expected solutions is illustrated in Fig. 1. In the left plot, a vehicle transports ordered items from a central depot to each purchaser separately and returns back to the origin for the next delivery task. This topology usually requires high operational costs; thus, a new routing topology in the right plot is proposed. The delivery services hire multiple vehicles to operate concurrently, where each vehicle can reach multiple customers in one route with an optimal operation cost. To evaluate the proposed approach, we implemented it in a real-world scenario, where delivery services were utilized to deliver goods to twenty six suburbs in Canberra, a capital city of Australia. The obtained results demonstrate effectiveness of the proposed method.

The remaining of the paper is arranged as follows. Section II introduces the DROP for multiple vehicles effectively used in goods delivery services by exploiting the mixed integer programming paradigm. Section III presents in detail how to employ the genetic algorithm to solve the mDROP problem. The proposed approach is then verified by a real-world goods delivery example in Canberra as discussed in Section IV before conclusions are drawn in Section V.

\section{PROBLEM STATEMENT}

In this work, we consider $m$ delivery vehicles that can be employed to deliver goods from a central depot to $n$ customers (also called nodes interchangeably). In order to effectively plan routes for the vehicles, we incorporate the following constraints into the mDROP problem.

- The first constraint requires that all routes start and finish at the depot.

- In the second constraint every single route consists of a specific set of customers, and all the routes are disjoint except depot node [15]. That is, goods for a specific customer can be delivered by only one delivery vehicle. However, those delivery vehicles may meet each other at the depot.

- Each delivery vehicle is constrained by its energy and capacity. In other words, a route for each vehicle is limited by a maximum distance.

Therefore, the optimization problem mDROP for finding the optimal routes for multiple delivery vehicles operating simultaneously aims to minimize the total distances of all the vehicle routes given their constraints. By exploiting the mixed integer programming model, the mDROP can be formulated as follows.

$$
\min \sum_{i=1}^{n} \sum_{j=1}^{n} c_{i j} x_{i j} .
$$

Subject to:

$$
\begin{gathered}
\sum_{j=2}^{n} x_{1, j}=m, \\
\sum_{j=2}^{n} x_{j, 1}=m, \\
\sum_{i=1}^{n} x_{i j}=1, \ldots, n, \\
\sum_{j=1}^{n} x_{i j}=1, \ldots, n, \\
\sum_{k=0}^{p_{k}} \sum_{j=0}^{p_{k}} c_{i j} x_{i j} \leq S_{e}, k=1, \ldots, m,
\end{gathered}
$$

where

$$
x_{i j} \in\{0,1\}
$$

is a binary number which is equal to 1 if it represents the path starting from a node $i$ and ending at a node $j$, otherwise it is equal to $0 . c_{i j}$ defines the distance from a node $i$ to a node $j$ while $S_{e}$ denotes the maximum distance a delivery vehicle can travel at its full energy budget. And $p_{k}$ is the number of customers the $k^{t h}$ needs to visit.

Mathematically, the mixed integer programming optimization problem can be addressed by an adopting heuristic approach [16], [17], [18], [19]. Nevertheless, in this work, we propose to employ the genetic algorithm with great advantage of the multi-chromosome mechanism to effectively solve the optimization problem. Detailed discussion of the proposed solution is presented in the following section.

\section{MULTI-VEHICLE ROUTING STRATEGY}

To demonstrate how to address the mixed integer programming based delivery vehicle routing optimization problem by utilizing the genetic algorithm, we consider a real-world scenario of delivering goods from a central depot to 26 suburb locations in Canberra, Australia. Locations of those suburbs can be seen on Google map as depicted in Fig. 2.

In practice, a ground delivery vehicle can travel between two locations, not following a straight line but a real road given the traffic infrastructure that can be obtained directly through Google Map. That is, it is straightforward to build a distance matrix whose element presents the road distance between any two suburb locations. Hence, given 26 suburb locations in the example, a symmetric distance matrix is developed, where its diagonal line is dominated by all zeros. An example of a distance matrix for 5 random suburb locations is demonstrated in Table I.

We then propose to exploit the multidimensional scaling algorithm to reconstruct the map. The rescaling algorithm can retain the relative relationship of the distance data in the original space to the greatest extent, and the rescaled data has better geometric demonstration. 26 suburb locations in the reconstructed map are now shown in Fig. 3. It can be seen that the reconstructed map is spatially different from the 


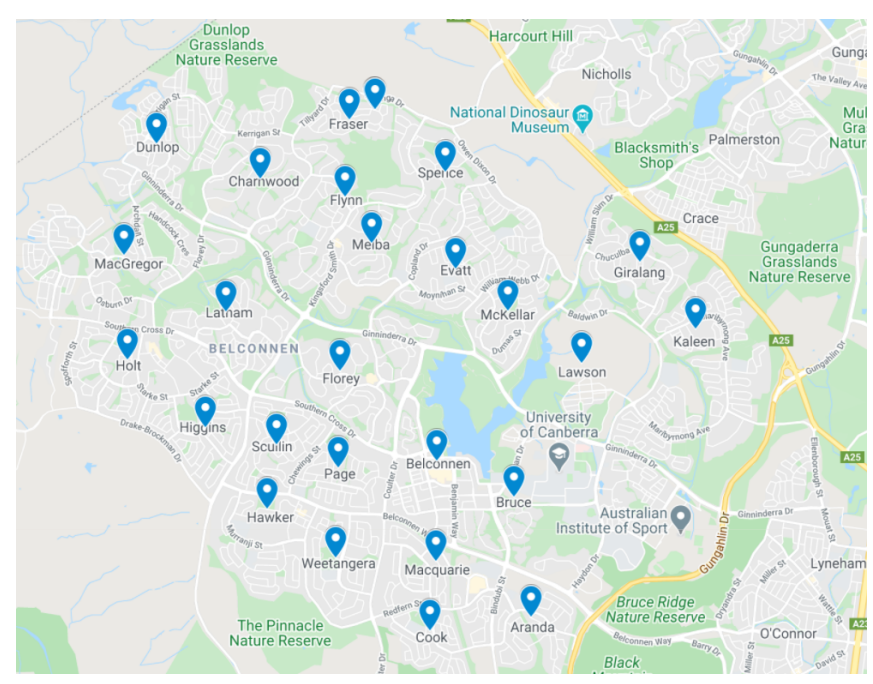

Fig. 2: Twenty six suburbs in Canberra, Australia depicted in Google map

TABLE I: AN EXAMPLE OF A DISTANCE MATRIX FOR 5 RANDOM SUBURB LOCATIONS

\begin{tabular}{|l|c|c|c|c|c|}
\hline & Aranda & Belconnen & Bruce & Chamwood & Cook \\
\hline Aranda & 0 & 3.9 & 2.1 & 9.3 & 2.4 \\
\hline Belconnen & 3.9 & 0 & 1.5 & 6.1 & 3 \\
\hline Bruce & 2.1 & 1.5 & 0 & 7.7 & 3 \\
\hline Chamwood & 9.3 & 6.1 & 7.7 & 0 & 8.6 \\
\hline Cook & 2.4 & 3 & 3 & 8.6 & 0 \\
\hline
\end{tabular}

real geological map as we only retain the relative distance information between any two suburb locations, which are needed for running the proposed algorithm.

Now we apply the genetic algorithm to solve the mDROP optimization problem (1) with constraints (2) - (6). At the beginning of the algorithm, the number of the vehicle routes is determined, corresponding to the number of chromosomes in the genetic algorithm. At the same time, we also need to determine the minimum number of nodes in each chromosome. The practical meaning of this setting is that when a delivery vehicle is hired by a merchant to carry out a delivery work, the delivery vehicle is required to complete of delivering goods to the minimum number of customers.

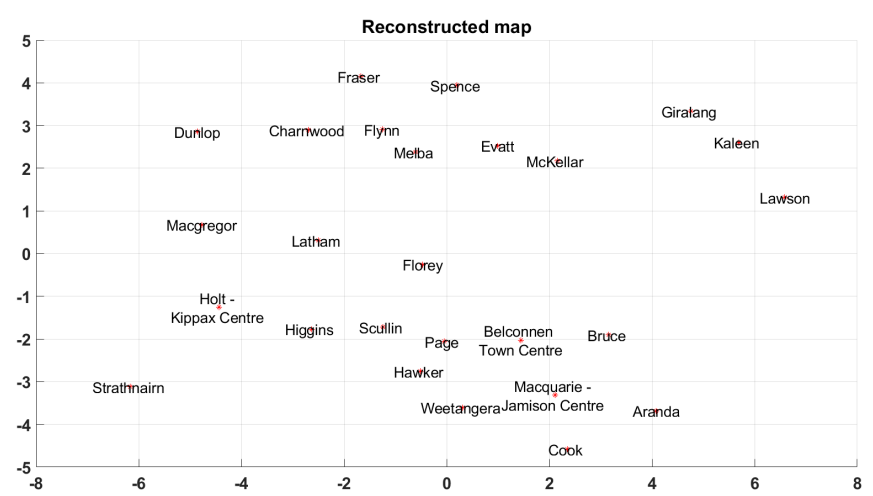

Fig. 3: Reconstructed map based on road distances

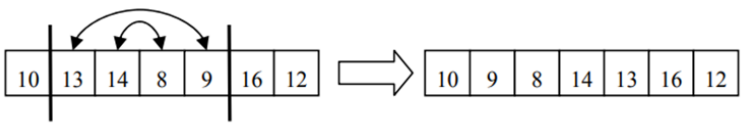

(a) Gene sequence inversion

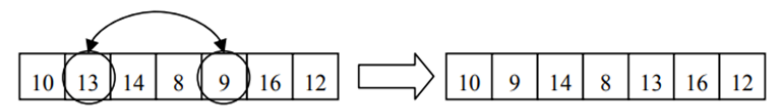

(b) Gene transportation

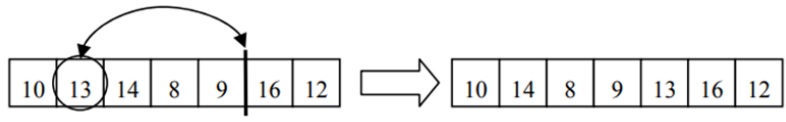

(c) Gene insertion

Fig. 4: Operations of the in-route mutation.

This is because the merchant needs to consider cost of employment; if it only hires a delivery vehicle to complete a delivery task for one or two customers, the transportation cost is too high. More importantly, each delivery vehicle is constrained by its energy; hence, a maximum distance threshold is set for each route the vehicle can travel.

Given the number of the vehicle routes, the proposed algorithm initiates a random path for each vehicle, which is similar to the number of the chromosomes randomly generated. The node order in the chromosome represents the order of each delivery destination. The order of each delivery location can only appear once in all the chromosomes and cannot be repeated. Subsequently, various treatments are performed on the nodes of the chromosomes, including the inroute and cross-route mutations. After each transformation, the total path length is calculated; and when the maximum number of the algorithm iterations is reached, the optimal solution is sorted in all the cases that have occurred.

More specifically, in the in-route mutation, selecting a random segment of a chromosome and reversing the order of its genes can be seen in Fig. 4a. The second types of the operations is to randomly select two gene sequences in the chromosome for sequence interchange as shown in Fig. $4 \mathrm{~b}$. On the other hand, a gene sequence can be randomly selected from the chromosome and inserted into other locations as illustrated in Fig. 4c. Likewise, there are several operations in the cross-route mutation. For instance, choosing two random parts from two chromosomes and swapping them can be conducted as shown in Fig. 5a. In addition, while two chromosomes can be combined together as demonstrated in Fig. 5b, they can be separated as illustrated in Fig. 5c.

In this work, we assume that the number of chromosomes in the genetic algorithm is constant. Moreover, since the two operations as shown in Figures $5 \mathrm{~b}$ and $5 \mathrm{c}$ result in a change in the chromosome order, we propose to use the first four gene sequences operations. It is noted that the delivery route must be a complete cycle; that is, the starting point is required to add to both the beginning and end of the sequence (chromosomes) when calculating the total path for each cycle. In the case that a certain number of the 


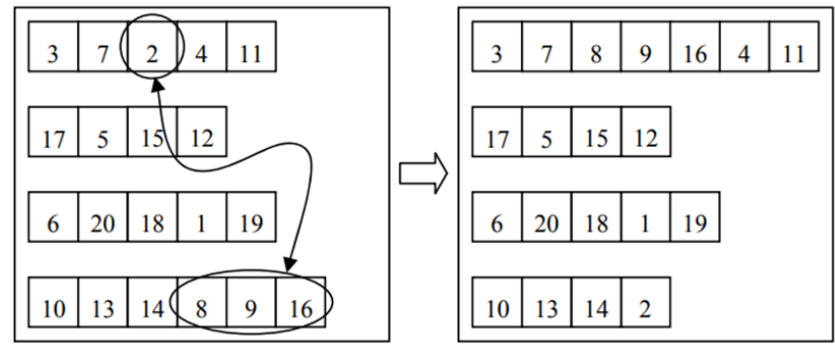

(a) Chromosome contraction

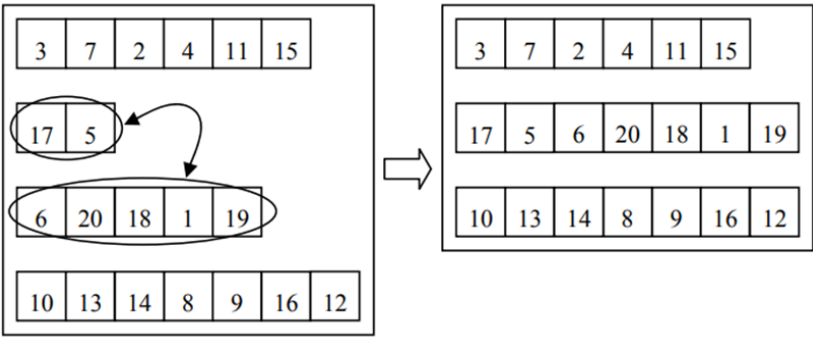

(b) Chromosome combination

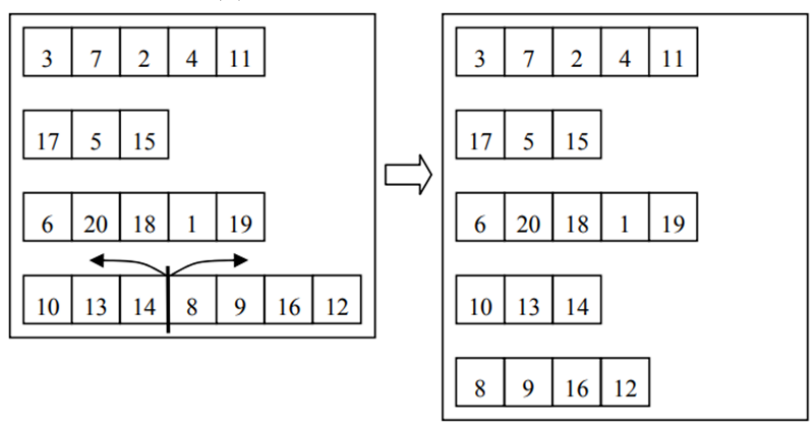

(c) Chromosome partition

Fig. 5: Operations of the cross-route mutation.

delivery vehicles is decided and the maximum number of the algorithm iterations is reached, if at least one vehicle is required to travel more than its energy allows, the number of the delivery vehicles must be increased. The procedure is repeated until the optimal solution is obtained.

\section{EXPERIMENTAL RESULTS AND DISCUSSIONS}

In order to demonstrate effectiveness of the proposed approach, we conducted the experiments using the electricityassisted cargo bicycles to deliver goods from one central depot to 26 suburb locations in Canberra as depicted in Fig. 2. It is assumed that each electricity-assisted cargo bicycle is equipped with a $480 \mathrm{Wh}$ battery and can provide a ride with around 19 miles $(\sim 30.6 \mathrm{~km})$ long per charge [20]. For the simplicity purpose, the vehicle's storage capacity and customers' ordered quantity are neglected. At the depot, it is expected to know the optimal number of delivery vehicles needed to effectively deliver goods to 26 suburb locations so that transportation cost can be minimized. It is noted that the depot was not located in the middle of the region.

At the starting point (i.e. depot), the distance travelled for each electric bicycle is initialized to zero. Every route must start from the depot, complete all the deliveries and finally back to the depot for recharging as well as restocking. Returning from the last route is considered as an indication of completing the delivery task. To specifically determine a set of customers visited by each electric bicycle in its particular delivery route considering its battery life constraint, we implemented the genetic algorithm to address the optimization problem (1) - (6). It is noted that each customer can be only visited by one delivery vehicle in the problem.

Regarding the other parameters in the proposed algorithm, we obtained the coordinates of 26 suburb locations on Google map, which were then used to compute their corresponding distance matrix. We also set the population size in the genetic algorithm to 80 while its maximum number of iterations was set to $7 \times 10^{3}$.

At beginning, we assumed that only one delivery vehicle is required to complete the task of delivering goods to 26 suburb locations. By running the proposed algorithm, we obtained the optimal routing path for the vehicle as demonstrated in Fig. 6a. Though the algorithm operated only 177 iterations to find the solution, the total travelling distance for the vehicle is about $50.4 \mathrm{~km}$, which is much longer than its battery capacity (i.e. $30.6 \mathrm{~km}$ ). We then increased the number of the electric bicycles needed to 2,3 , and 4 , and run the planning algorithm again. The optimal route paths for those vehicles obtained by the algorithm are demonstrated in Figures $6 \mathrm{~b}, 6 \mathrm{c}$ and $6 \mathrm{~d}$, respectively. Nonetheless, those 3 scenarios, the maximum route at least one electricityassisted cargo bike required to ride is still longer than the distance it can actually travel. The algorithm rerun with 5 electric bikes assumed to be used in the delivery task. The obtained results show that the maximum distance one electric vehicle is expected to run in this scenario is within its battery constraint. The rout planning algorithm stopped at 5 electric bicycles and their optimal resulting routes are illustrated in Fig. 7c. It is noted that the proposed algorithm required only 229 iterations to find the near-optimal routing paths for the delivery vehicles, where the total length of the routes is about $70.3 \mathrm{~km}$. However, we kept running the algorithm and it converged to a stationary solution after about 3800 iterations where where the total length of the routes reaches a constant of $68.9305 \mathrm{~km}$, as illustrated in Fig. 7d. The small error of $1.95 \%$ between near-optimal and stationary solutions promises practicality of the proposed method in real-time systems.

\section{CONCLUSIONS}

The paper has presented an efficient approach to design the optimal routes for multiple ground delivery vehicles given their energy constraint in order to minimize transportation cost in goods delivery services. It is proposed to employ multiple delivery vehicles so that goods can be delivered to customers on time. Nevertheless, the problem becomes beyond a traditional TSP problem. Therefore, the paper has proposed to exploit the mixed integer programming model to formulate the delivery multi-vehicle routing optimization problem with a constraint of the vehicle energy capacity. It 


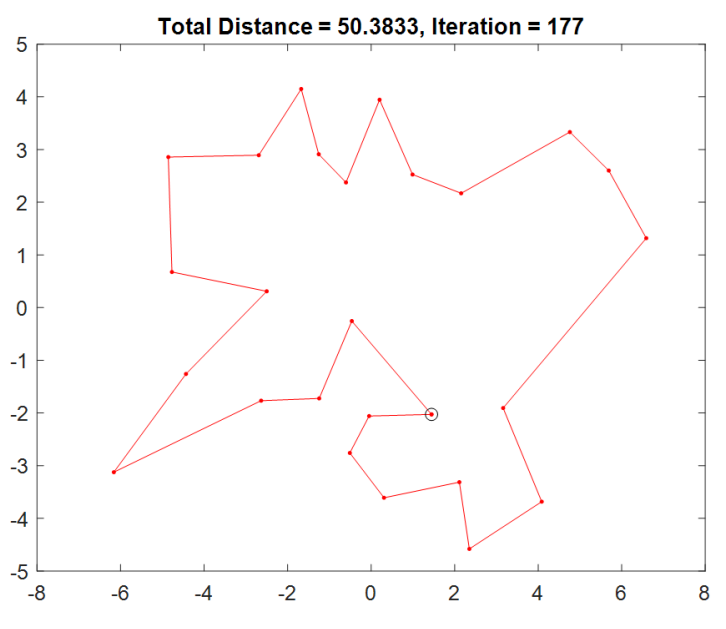

(a) The optimal route for one delivery vehicle.

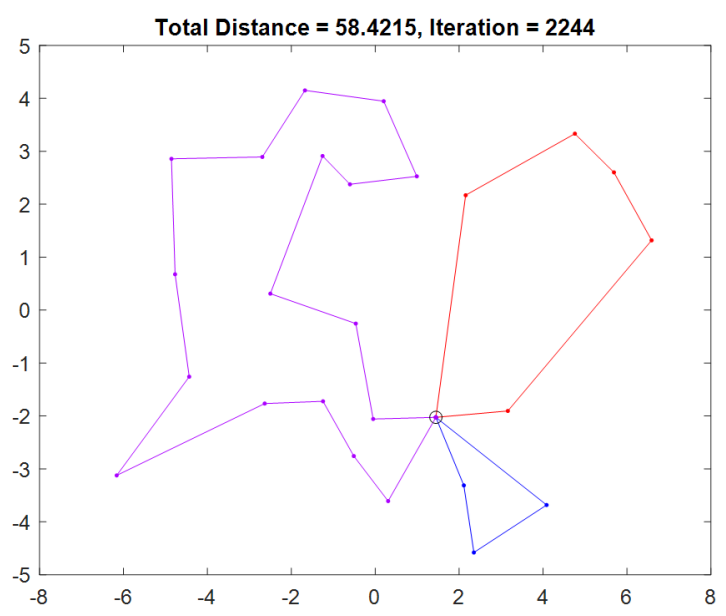

(c) The optimal routes for three delivery vehicles

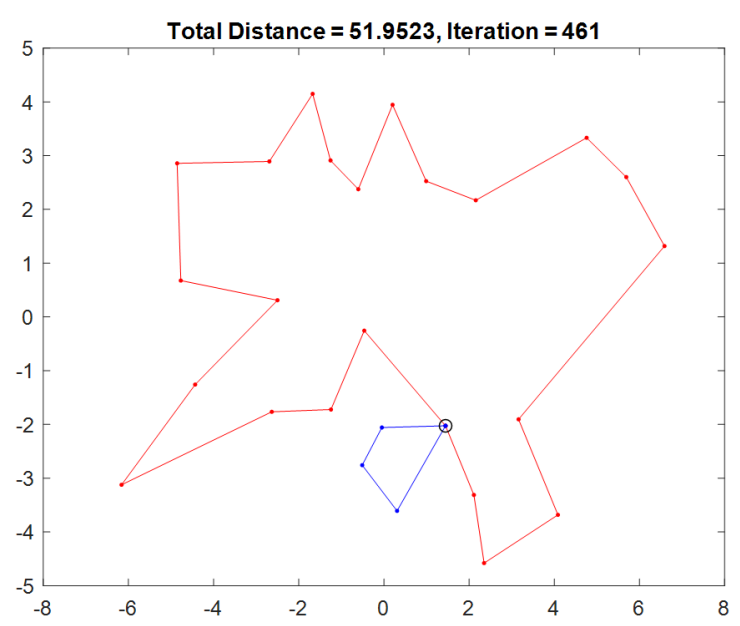

(b) The optimal routes for two delivery vehicles.

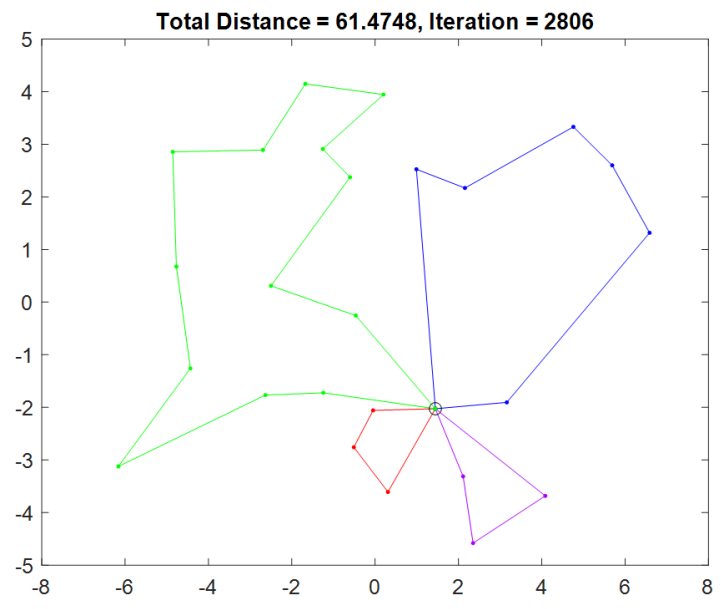

(d) The optimal routes for four delivery vehicles

Fig. 6: The optimal routes for delivering goods in 26 suburb locations in Canberra using (a) one delivery vehicle, (b) two delivery vehicles, (c) three delivery vehicles and (d) four delivery vehicles.

is also noted that the multi-vehicle routes are disjoint except the depot node. In order to effectively solve the routing optimization problem, it has been proposed to employ the multi-chromosome genetic algorithm, which can optimize the number of delivery vehicles needed as well. The proposed method was implemented in a real-life experiment where it was required to deliver goods from a depot to 26 suburb locations in Canberra using the electric bikes. Given the bicycle battery capacity, the algorithm returned 5 electric bicycles needed to complete the delivery task, where their routing paths were also optimized. The obtained results show practicality of the proposed technique.

\section{REFERENCES}

[1] P. Dannenberg, M. Fuchs, T. Riedler and C. Wiedemann, "Digital Transition by COVID-19 Pandemic? The German Food Online Retail," Tijdschrift voor Economische en Sociale Geografie, Vol. 111, No. 3, pp. 543-560, 2020. DOI:10.1111/tesg. 12453.

[2] R. H. Ballou, "The evolution and future of logistics and supply chain management,", European Business Review, vol. 19, no. 4, pp. 332-348, 2007.
[3] A. Buchegger, K. Lassnig, S. Loigge, C. Mühlbacher and G. Steinbauer, "An Autonomous Vehicle for Parcel Delivery in Urban Areas," 2018 21st International Conference on Intelligent Transportation Systems (ITSC), Maui, HI, 2018, pp. 2961-2967, doi: 10.1109/ITSC.2018.8569339.

[4] K. Dorling, J. Heinrichs, G. G. Messier and S. Magierowski, "Vehicle Routing Problems for Drone Delivery," in IEEE Transactions on Systems, Man, and Cybernetics: Systems, vol. 47, no. 1, pp. 70-85, Jan. 2017, doi: 10.1109/TSMC.2016.2582745.

[5] F. Schenkelberg, "How reliable does a delivery drone have to be?," 2016 Annual Reliability and Maintainability Symposium (RAMS), Tucson, AZ, USA, 2016, pp. 1-5, doi: 10.1109/RAMS.2016.7448054.

[6] M. Sheth, P. Butrina, A. Goodchild, and E. McCormack, "Measuring delivery route cost trade-offs between electric-assist cargo bicycles and delivery trucks in dense urban areas," European Transport Research Review, vol. 11, no. 1, pp. 1-12, Dec 2019.

[7] L. Richardson, "Platforms, Markets, and Contingent Calculation: The Flexible Arrangement of the Delivered Meal," Antipode, vol. 52, no. 3, pp. 619-636, 2020/05/01 2020, doi: 10.1111/anti.12546.

[8] L. Nguyen, G. Hu and C. J. Spanos, "Efficient spatio-temporal sensor deployments: A smart building application," 2017 13th IEEE International Conference on Control Automation (ICCA), 2017, pp. 612-617. 


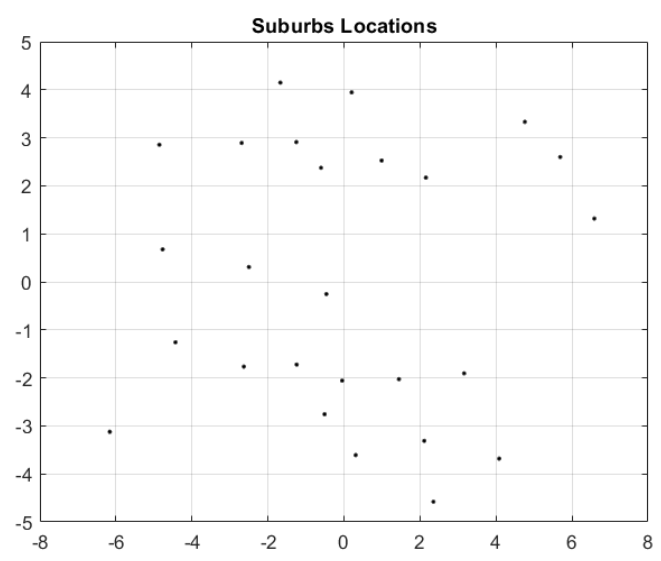

(a) 26 suburb locations.

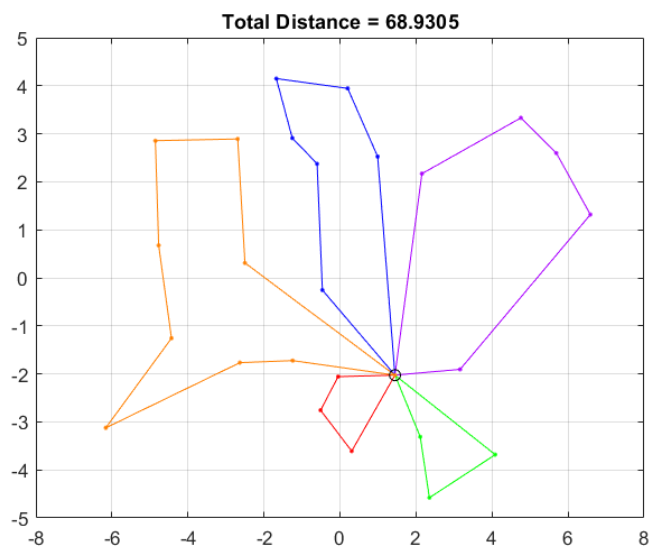

(c) The optimal routes for five delivery vehicles

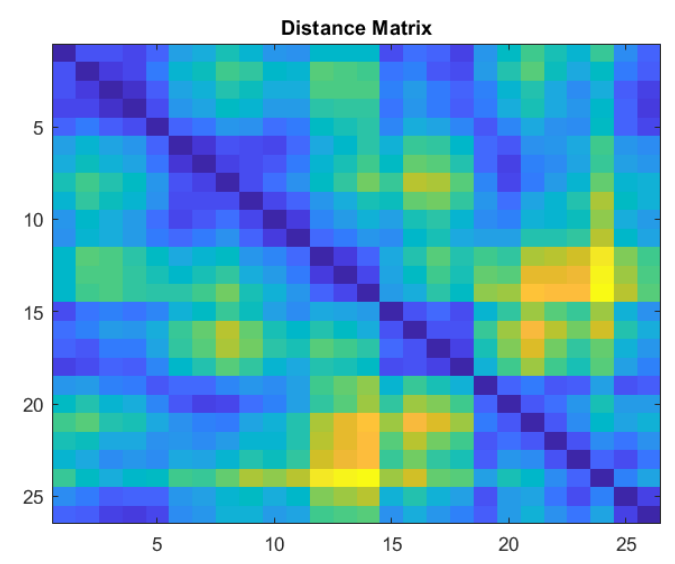

(b) The distance matrix.

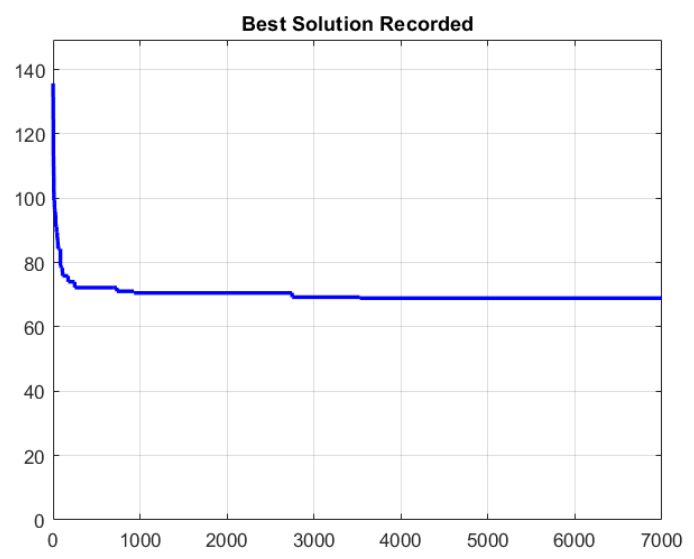

(d) The shortest total length of the travelling distances

Fig. 7: The optimal strategy for delivering goods in 26 suburb locations in Canberra using five delivery vehicles.

[9] L. Nguyen, G. Hu and C. J. Spanos, "Efficient Sensor Deployments for Spatio-Temporal Environmental Monitoring," IEEE Transactions on Systems, Man, and Cybernetics: Systems, vol. 50, no. 12, pp. 53065316, doi: 10.1109/TSMC.2018.2872041.

[10] P. Miliotis. "Using cutting planes to solve the symmetric travelling salesman problem. Mathematical Programming," 15(1):177-188, 1978.

[11] S. Basu, "Tabu search implementation on traveling salesman problem and its variations: A literature survey," Am. J. Oper. Res., vol. 02, no. 02, pp. 163-173, 2012.

[12] R. Baldacci, E. Hadjiconstantinou, and A. Mingozzi, "An exact algorithm for the capacitated vehicle routing problem based on a twocommodity network flow formulation," Oper. Res., vol. 52, no. 5, pp. 723-738, 2004.

[13] Y. Hu, Z. Zhang, Y. Yao, X. Huyan, X. Zhou, and W. S. Lee, "A bidirectional graph neural network for traveling salesman problems on arbitrary symmetric graphs," Eng. Appl. Artif. Intell., vol. 97, no. 104061, p. 104061, 2021.

[14] M. Gen and R. Cheng. "Genetic algorithms and engineering design," Wiley-Interscience, 1997.

[15] P. Kitjacharoenchai, M. Ventresca, M. Moshref-Javadi, S. Lee, J. M. A Tanchoco, and P. A. Brunese, "Multiple travelling salesman problem with drones: Mathematical model and heuristic approach," Comput. Ind. Eng., vol. 129, pp. 14-30, 2019.

[16] A. Király and J. Abonyi, "Optimization of Multiple Traveling Salesmen Problem by a Novel Representation Based Genetic Algorithm,' in Intelligent Computational Optimization in Engineering: Techniques and Applications, M. Koppen, G. Schaefer, and A. Abraham Eds. Berlin, Heidelberg: Springer Berlin Heidelberg, 2011, pp. 241-269.

[17] A. Király and J. Abonyi, "Redesign of the Supply of Mobile Mechanics based on a novel Genetic Optimization Algorithm using Google Maps API," Engineering Applications of Artificial Intelligence, vol. 38, 11/20 2014, doi: 10.1016/j.engappai.2014.10.015.

[18] L. Nguyen, N. Ulapane and J. Valls Miro, "Adaptive sampling for spatial prediction in environmental monitoring using wireless sensor networks: A review," 2018 13th IEEE Conference on Industrial Electronics and Applications (ICIEA), 2018, pp. 346-351.

[19] L. Nguyen, S. Kodagoda, R. Ranasinghe, and G. Dissanayake, "Mobile Robotic Sensors for Environmental Monitoring using Gaussian Markov Random Field," Robotica, vol. 39, no. 5, pp. 862-884, doi: 10.1017/S026357472000079X.

[20] M. Toll. "The truth: How far can an electric bicycle really go on a single charge?" https://electrek.co/2020/06/12/how-far-can-an-electricbicycle-really-go-on-a-charge/ (accessed 10 Feb, 2021). 